\title{
LINEAR CONNECTIONS AND EXTENDED ELECTRODYNAMICS
}

\author{
STOIL DONEV AND MARIA TASHKOVA
}

Communicated by Ivaïlo M. Mladenov

Abstract. In this paper we give a presentation of the basic vacuum relations of Extended Electrodynamics in terms of linear connections.

\section{Linear Connections}

Linear connections are first-order differential operators in vector bundles. If such a connection $\nabla$ is given and $\sigma$ is a section of the bundle, then $\nabla \sigma$ is oneform on the base space valued in the space of sections of the vector bundle, so if $X$ is a vector field on the base space then $i(X) \nabla \sigma=\nabla_{X} \sigma$ is a new section of the same bundle [2]. If $f$ is a smooth function on the base space then $\nabla(f \sigma)=\mathrm{d} f \otimes \sigma+f \nabla \sigma$, which justifies the differential operator nature of $\nabla$ : the components of $\sigma$ are differentiated and the basis vectors in the bundle space are linearly transformed.

Let $e_{a}$ and $\varepsilon^{b}, a, b=1,2, \ldots, r$ be two dual local bases of the corresponding spaces of sections $<\varepsilon^{b}, e_{a}>=\delta_{a}^{b}$, then we can write

$\sigma=\sigma^{a} e_{a}, \quad \nabla=\mathbf{d} \otimes \mathrm{id}+\Gamma_{\mu a}^{b} \mathrm{~d} x^{\mu} \otimes\left(\varepsilon^{a} \otimes e_{b}\right), \quad \nabla\left(e_{a}\right)=\Gamma_{\mu a}^{b} \mathrm{~d} x^{\mu} \otimes e_{b}$

and therefore

$\nabla\left(\sigma^{m} e_{m}\right)=\mathbf{d} \sigma^{m} \otimes e_{m}+\sigma^{m} \Gamma_{\mu a}^{b} \mathrm{~d} x^{\mu}<\varepsilon^{a}, e_{m}>\otimes e_{b}=\left[\mathbf{d} \sigma^{b}+\sigma^{a} \Gamma_{\mu a}^{b} \mathrm{~d} x^{\mu}\right] \otimes e_{b}$

where $\Gamma_{\mu a}^{b}$ are the components of $\nabla$ with respect to the coordinates $\left\{x^{\mu}\right\}$ on the base space and with respect to the bases $\left\{e_{a}\right\}$ and $\left\{\varepsilon^{b}\right\}$.

Since the elements $\left(\varepsilon^{a} \otimes e_{b}\right)$ define a basis of the space of (local) linear maps of the local sections, it becomes clear that in order to define locally a linear connection it is sufficient to specify some one-form $\theta$ on the base space and a 
linear map

$\phi=\phi_{a}^{b} \varepsilon^{a} \otimes e_{b}$ in the space of sections. Then

$$
\nabla(\sigma)=\mathbf{d} \sigma^{a} \otimes e_{a}+\theta \otimes \phi(\sigma)
$$

defines a linear connection with components $\Gamma_{\mu a}^{b}=\theta_{\mu} \phi_{a}^{b}$ in these bases. So, locally, any linear connection $\nabla$ can be written as

$$
\nabla=\mathbf{d} \otimes\left(\varepsilon^{a} \otimes e_{a}\right)+\Psi_{\mu a}^{b} \mathrm{~d} x^{\mu} \otimes\left(\varepsilon^{a} \otimes e_{b}\right) .
$$

Let $\Psi_{1}$ and $\Psi_{2}$ be two one-forms valued in the space of linear maps in a vector bundle. A map $\left(\Psi_{1}, \Psi_{2}\right) \rightarrow(\wedge, \odot)\left(\Psi_{1}, \Psi_{2}\right)$ is defined by (we shall write just $\odot$ for $(\wedge, \odot)$ and the usual $\circ$ will mean just composition)

$$
\begin{aligned}
\odot\left(\Psi_{1}, \Psi_{2}\right) & =\left(\Psi_{1}\right)_{\mu a}^{b}\left(\Psi_{2}\right)_{\nu m}^{n} \mathrm{~d} x^{\mu} \wedge \mathrm{d} x^{\nu} \otimes\left[\circ\left(\varepsilon^{a} \otimes e_{b}, \varepsilon^{m} \otimes e_{n}\right)\right] \\
& =\left(\Psi_{1}\right)_{\mu a}^{b}\left(\Psi_{2}\right)_{\nu m}^{n} \mathrm{~d} x^{\mu} \wedge \mathrm{d} x^{\nu} \otimes\left[<\varepsilon^{a}, e_{n}>\left(\varepsilon^{m} \otimes e_{b}\right)\right] \\
& =\left(\Psi_{1}\right)_{\mu a}^{b}\left(\Psi_{2}\right)_{\nu m}^{a} \mathrm{~d} x^{\mu} \wedge \mathrm{d} x^{\nu} \otimes\left(\varepsilon^{m} \otimes e_{b}\right) \mu<\nu .
\end{aligned}
$$

In the case of trivial vector bundles, the curvature of $\nabla$ is given by [2]

$$
\left[\mathbf{d}\left(\Psi_{\mu a}^{b} \mathrm{~d} x^{\mu}\right)\right] \otimes\left(\varepsilon^{a} \otimes e_{a}\right)+\odot(\Psi, \Psi) .
$$

\section{Some Facts From the Clasical and the Extended Electrodynamics}

We recall now some facts from the Classical Electrodynamics (CED) and from the Extended Electrodynamics (EED) [1]. The vector bundle under consideration is the (trivial) bundle $\Lambda^{2}(M)$ of two-forms on the Minkowski space-time $M$. Recall that if $(F, * F)$ is a CED vacuum solution, i.e., $\mathbf{d} F=0, \mathbf{d} * F=0$, then the combinations

$$
\mathcal{F}=a F-b * F, \quad \mathcal{F}^{*}=b F+a * F
$$

where $(a, b)$ are two arbitrary real numbers, also give a CED vacuum solution and, since on Minkowski space the corresponding Hodge star $*_{2}$ satisfies the relation $*^{2}=-\mathrm{id}_{\Lambda^{2}(M)}$, we obtain $\mathcal{F}^{*}=* \mathcal{F}$. The two corresponding energy tensors are related by

$$
T\left(\mathcal{F}, \mathcal{F}^{*}\right)=\left(a^{2}+b^{2}\right) T(F, * F) .
$$

Recall the real representation of complex numbers $z=a I+b J$ where $I$ is the unit matrix in $\mathbb{R}^{2}$ and $J$ is the standard complex structure matrix in $\mathbb{R}^{2}$ with 
columns $(0,-1)^{T},(1,0)^{T}$. So, we obtain an action of the linear group $G$ of matrices $\alpha=a I+b J$ on the CED vacuum solutions. This is a commutative group $G$ and its Lie algebra $\mathcal{G}$ just adds the zero $(2 \times 2)$ matrix to $G$, and $(I, J)$ define a natural basis of $\mathcal{G}$. So, having a CED vacuum solution, we have in fact a two-parameter family of vacuum solutions. Hence, we can define a $\mathcal{G}$-valued two-form $\Omega$ on $M$ by $\Omega=F \otimes I+* F \otimes J$, and the equation $\mathbf{d} \Omega=0$ is equivalent to $\mathbf{d} F=0, \mathbf{d} * F=0$.

Consider the new basis $\left(I^{\prime}, J^{\prime}\right)$ in $\mathcal{G}$ given by

$$
I^{\prime}=a I+b J, \quad J^{\prime}=-b I+a J .
$$

Accordingly, the "new" solution $\Omega^{\prime}$, i.e., the old solution in the new basis of $\mathcal{G}$, will be

$$
\begin{aligned}
\Omega^{\prime} & =F \otimes I^{\prime}+* F \otimes J^{\prime}=F \otimes(a I+b J)+* F \otimes(-b I+a J) \\
& =(a F-b * F) \otimes I+(b F+a * F) \otimes J \mathcal{F} \otimes I+\mathcal{F}^{*} \otimes J
\end{aligned}
$$

In view of this we may consider this transformation as nonessential, i.e., we may consider $(F, * F)$ and $\left(\mathcal{F}, \mathcal{F}^{*}\right)$ as two different representations in corresponding bases of $\mathcal{G}$ of the same solution.

Such an interpretation is approporiate and useful if the field shows some invariant properties with respect to this class of transformations. For example, if the Lorentz invariants

$$
I_{1}=\frac{1}{2} F_{\mu \nu} F^{\mu \nu}=\left(\mathbf{B}^{2}-\mathbf{E}^{2}\right), \quad I_{2}=\frac{1}{2} F_{\mu \nu}(* F)^{\mu \nu}=2 \mathbf{E} . \mathbf{B}
$$

where $\mathbf{E}$ and $\mathbf{B}$ are the corresponding electric and magnetic components of $F$, are zero: $I_{1}=I_{2}=0$, (the so called "null field case") then all the above transformations keep unchanged these zero-values of $I_{1}$ and $I_{2}$. In fact, under such a transformation $(F, * F) \rightarrow\left(\mathcal{F}, \mathcal{F}^{*}\right)$ the two Lorentz invariants transform to $\left(I_{1}^{\prime}, I_{2}^{\prime}\right)$ in the following way

$$
I_{1}^{\prime}=\left(a^{2}-b^{2}\right) I_{1}+2 a b I_{2}, \quad I_{2}^{\prime}=-2 a b I_{1}+\left(a^{2}-b^{2}\right) I_{2}
$$

and the determinant of this transformation is $\left(a^{2}+b^{2}\right)^{2} \neq 0$. So, a null field, i.e., a field with zero invariants $I_{1}$ and $I_{2}$, stays a null field under these transformations. Moreover, NO non-null field can be transformed to a null field by means of these transformations, and, conversely, NO null field can be transformed to a non-null field in this way. Hence, the Lorentz invariance and the dual $G$-invariance of $I_{1}$ and $I_{2}$ hold simultanoiusly only in the null-field case. 
Further we are going to pay due respect to this invariance, keeping in mind the basic fact that only in this case the velocity of the energy propagation of the field is equal to "c" and follows straight lines, so this is intrinsic property of the field. In order to come to the equations of EED we can recall that every bilinear map $\varphi: \mathcal{G} \times \mathcal{G} \rightarrow W$, where $W$ is some linear space with basis $\left\{e_{i}\right\}, i=1,2, \ldots$, defines corresponding product in the $\mathcal{G}$-valued differential forms by means of the relation

$$
\varphi\left(\Omega_{1}^{i} \otimes e_{i}, \Omega_{2}^{j} \otimes e_{j}\right)=\Omega_{1}^{i} \wedge \Omega_{2}^{j} \otimes \varphi\left(e_{i}, e_{j}\right) .
$$

Recall now the following identity in Minkowski space

$$
I_{1} \delta_{\mu}^{\nu} \equiv \frac{1}{2} F_{\alpha \beta} F^{\alpha \beta} \delta_{\mu}^{\nu}=F_{\sigma \mu} F^{\sigma \nu}-(* F)_{\sigma \mu}(* F)^{\sigma \nu}
$$

and the standard energy-tensor $Q_{\mu}^{\nu}$ of electromagnetic field

$$
Q_{\mu}^{\nu}=-\frac{1}{2}\left[F_{\sigma \mu} F^{\sigma \nu}+(* F)_{\sigma \mu}(* F)^{\sigma \nu}\right] .
$$

We see that under $I_{1}=0$ the two fields $F$ and $* F$ carry the same energymomentum during propagation. Moreover, there is NO interaction stress-energymomentum between $F$ and $* F$ as it is seen from the expression for $Q_{\mu}^{\nu}$.

Corollary 1. The two fields $F$ and $* F$ may interact only in regime of dynamical equilibrium, i.e., any energy-momentum loss of $F / * F$ should be compensated by equal gain of $* F / F: F \rightleftarrows * F$.

EED makes use of these facts assuming that the corresponding dynamical equations must have local energy-momentum exchange physical sense, so the symmetry $F \rightleftarrows * F$ must be respected.

Now, let $\varphi=\vee$, where " $\vee$ " is the symmetrized tensor product in $\mathcal{G}$. We consider the expression $\vee(\Omega, * \mathbf{d} \Omega)$.

$$
\begin{aligned}
\vee(\Omega, * \mathbf{d} \Omega)= & (F \wedge * \mathbf{d} F) \otimes I \vee I(* F \wedge * \mathbf{d} * F) \otimes J \vee J \\
& +(F \wedge * \mathbf{d} * F \otimes+* F \wedge * \mathbf{d} F) \otimes I \vee J .
\end{aligned}
$$

The vacuum EED equations are $\vee(\Omega, * \mathbf{d} \Omega)=0$, or equivalently

$$
F \wedge * \mathbf{d} F=0, \quad(* F) \wedge * \mathbf{d} * F=0, \quad F \wedge * \mathbf{d} * F+(* F) \wedge * \mathbf{d} F=0 .
$$

In terms of the codifferential $\delta=* \mathbf{d} *$ these equations look like

$$
\delta * F \wedge F=0, \quad \delta F \wedge * F=0, \quad \delta F \wedge F-\delta * F \wedge * F=0 .
$$


Correspondingly in components we obtain

$$
\begin{aligned}
\frac{1}{2} F^{\alpha \beta}(\mathbf{d} F)_{\alpha \beta \mu} & \equiv(* F)_{\mu \nu}(\delta * F)^{\nu}=0 \\
\frac{1}{2}(* F)^{\alpha \beta}(\mathbf{d} * F)_{\alpha \beta \mu} & \equiv F_{\mu \nu}(\delta F)^{\nu}=0 \\
\frac{1}{2}(* F)^{\alpha \beta}(\mathbf{d} F)_{\alpha \beta \mu}+\frac{1}{2} F^{\alpha \beta}(\mathbf{d} * F)_{\alpha \beta \mu} & \equiv(\delta * F)^{\nu} F_{\nu \mu}+(\delta F)^{\nu}(* F)_{\nu \mu}=0 .
\end{aligned}
$$

\section{Basic Property of the Nonlinear Solutions}

All nonlinear solutions to the EED vacuum equations, namely, those satisfying $\mathbf{d} F \neq 0, \mathbf{d} * F \neq 0$, have zero invariants: $I_{1}=I_{2}=0$, so, they minimize the quantity $I_{1}^{2}+I_{2}^{2} \geq 0$. Moreover, for any nonlinear solution defined by $F$ there exists a canonical coordinate system on $M$, called further $F$-adapted, in which $F$ and $* F$ look as follows

$$
\begin{gathered}
F=A \wedge \zeta, \quad * F=A^{*} \wedge \zeta \\
A=u \mathrm{~d} x+p \mathrm{~d} y, \quad A^{*}=p \mathrm{~d} y-u \mathrm{~d} z, \quad \zeta=\varepsilon \mathrm{d} z+\mathrm{d} \xi, \quad \varepsilon \pm 1
\end{gathered}
$$

and $(u, p)$ are two functions on $M$, satisfying the equation

$$
u\left(u_{\xi}-\varepsilon u_{z}\right)+p\left(p_{\xi}-\varepsilon p_{z}\right)=0 .
$$

As for the energy-momentum tensor $T_{\mu \nu}$ of the vacuum solutions, considered as a symmetric two-form on $M$, it is defined in terms of $\Omega$ as follows

$$
\begin{aligned}
T(X, Y) & =\frac{1}{2} * g[i(X) \Omega, * i(Y) \Omega] \\
& =-\frac{1}{2} X^{\mu} Y^{\nu}\left[F_{\mu \sigma} F_{\nu}{ }^{\sigma}+(* F)_{\mu \sigma}(* F)_{\nu}{ }^{\sigma}\right]=X^{\mu} Y^{\nu} T_{\mu \nu}
\end{aligned}
$$

where $(X, Y)$ are two arbitrary vector fields on $M, g$ is the metric in $\mathcal{G}$ defined by $g(\alpha, \beta)=\frac{1}{2} \operatorname{tr}\left(\alpha \cdot \beta^{*}\right)$, and $\beta^{*}$ is the transposed to $\beta$. Note that $g(I, J)=0$, which elliminates the corresponding coefficient which reads

$$
F_{\mu \sigma}(* F)^{\nu \sigma}+(* F)_{\mu \sigma} F^{\nu \sigma}=\frac{1}{2} F_{\alpha \beta}(* F)^{\alpha \beta} \delta_{\mu}^{\nu}
$$

so, in a $g$-NONorthogonal basis of $\mathcal{G}$ this coefficient will appear. 
Finally, recall the generalization of Lie derivative $\mathcal{L}_{K}$ with respect to the $k$ vector $K$, acting in the exterior algebra of differential forms according to the formula $\mathcal{L}_{K}=i(K) \mathbf{d}-(-1)^{k} \mathbf{d} i(K)$. Then, in view of the relations $F_{\mu \nu} F^{\mu \nu}=(* F)_{\mu \nu} F^{\mu \nu}=0$, the above equations acquire the form

$$
\mathcal{L}_{\bar{F}} F=0, \quad \mathcal{L}_{* \bar{F}}(* F)=0, \quad \mathcal{L}_{\bar{F}}(* F)+\mathcal{L}_{(* \bar{F})} F=0
$$

where $\bar{F}$ and $* \bar{F}$ are the $\eta$-corresponding two-vectors. In terms of the two-form $\Omega$ and

$\bar{\Omega}=\bar{F} \otimes e_{1}+* \bar{F} \otimes e_{2}$ these three equations can be united in one as follows $\mathcal{L}_{\bar{\Omega}}^{\vee} \Omega=\mathcal{L}_{\bar{F}} F \otimes e_{1} \vee e_{1}+\mathcal{L}_{* \bar{F}} * F \otimes e_{2} \vee e_{2}+\left(\mathcal{L}_{\bar{F}} * F+\mathcal{L}_{* \bar{F}} F\right) \otimes e_{1} \vee e_{2}=0$.

\section{Linear Connection Interpretation of the Nonlinear Part of the EED}

If $\mathcal{I}$ is the identity in $\Lambda^{2}(M)$ and $\mathcal{J}=*$ is the complex structure in $\Lambda^{2}(M)$ then a representation $\rho$ of $G$ in $\Lambda^{2}(M)$ is given by

$$
\rho(\alpha)=a \mathcal{I}+b \mathcal{J} .
$$

Also, a representation $\rho^{\prime}$ of the corresponding Lie algebra $\mathcal{G}$ is defined by the same relation. So, if $\alpha: M \rightarrow \mathcal{G}$ is a map then $\rho^{\prime}(\alpha)$ is a linear map in $\Lambda^{2}(M)$, and recalling our one-form $\zeta=\varepsilon \mathrm{d} z+\mathrm{d} \xi$ we define a linear connection $\nabla$ in $\Lambda^{2}(M)$ by

$$
\begin{aligned}
\nabla & =\mathbf{d} \otimes \operatorname{id}_{\Lambda^{2}(M)}+\zeta \otimes \rho^{\prime}(\alpha(u, p)) \\
& =\mathbf{d} \otimes \operatorname{id}_{\Lambda^{2}(M)}+\zeta \otimes(u \mathcal{I}+p \mathcal{J}), \quad \alpha \in \mathcal{G} .
\end{aligned}
$$

Two other connections $\bar{\nabla}$ and $\nabla^{*}$ are defined by

$$
\begin{aligned}
\rho^{\prime}(\bar{\alpha}(u, p)) & =\rho^{\prime}(\alpha(u,-p))=u \mathcal{I}-p \mathcal{J} \\
\left(\rho^{\prime}\right)^{*}(\alpha(u, p)) & =\rho^{\prime}(\alpha . J)=\rho^{\prime}(\alpha(-p, u))=-p \mathcal{I}+u \mathcal{J}
\end{aligned}
$$

and we introduce for further use

$$
\chi=u \mathcal{I}+p \mathcal{J}, \quad \bar{\chi}=u \mathcal{I}-p \mathcal{J}, \quad \chi^{*}=-p \mathcal{I}+u \mathcal{J} .
$$

Denoting

$$
\Psi=\zeta \otimes \chi, \quad \bar{\Psi}=\zeta \otimes \bar{\chi}, \quad \Psi^{*}=\zeta \otimes \chi^{*}
$$


we have (because $\zeta \wedge \zeta=0$ )

$$
\odot(\Psi, \Psi)=\odot(\Psi, \bar{\Psi})=\odot\left(\Psi, \Psi^{*}\right)=0
$$

Now, since

$$
\begin{aligned}
\Psi & =u \zeta \otimes \mathcal{I}+p \zeta \otimes \mathcal{J} \\
\bar{\Psi} & =u \zeta \otimes \mathcal{I}-p \zeta \otimes \mathcal{J} \\
\Psi^{*} & =-p \zeta \otimes \mathcal{I}+u \zeta \otimes \mathcal{J}
\end{aligned}
$$

we obtain for the corresponding curvatures

$$
\begin{aligned}
& \mathcal{R}=\mathbf{d}(u \zeta) \otimes \mathcal{I}+\mathbf{d}(p \zeta) \otimes \mathcal{J} \\
& \overline{\mathcal{R}}=\mathbf{d}(u \zeta) \otimes \mathcal{I}-\mathbf{d}(p \zeta) \otimes \mathcal{J} \\
& \mathcal{R}^{*}=\mathbf{d}(-p \zeta) \otimes \mathcal{I}+\mathbf{d}(u \zeta) \otimes \mathcal{J} .
\end{aligned}
$$

By direct calculation we obtain also

$$
\begin{aligned}
* \frac{1}{6} \operatorname{Tr}[\odot(\bar{\Psi}, * \mathbf{d} \Psi)]= & -\varepsilon\left[u\left(u_{\xi}-\varepsilon u_{z}\right)+p\left(p_{\xi}-\varepsilon p_{z}\right)\right] \mathrm{d} z \\
& -\left[u\left(u_{\xi}-\varepsilon u_{z}\right)+p\left(p_{\xi}-\varepsilon p_{z}\right)\right] \mathrm{d} \xi \\
\frac{1}{6} \operatorname{Tr}\left[\odot\left(\Psi^{*}, * \mathbf{d} \Psi\right)\right]= & \varepsilon\left[p\left(u_{\xi}-\varepsilon u_{z}\right)-u\left(p_{\xi}-\varepsilon p_{z}\right)\right] \mathrm{d} x \wedge \mathrm{d} y \wedge \mathrm{d} z \\
& +\left[p\left(u_{\xi}-\varepsilon u_{z}\right)-u\left(p_{\xi}-\varepsilon p_{z}\right)\right] \mathrm{d} x \wedge \mathrm{d} y \wedge \mathrm{d} \xi \\
= & \delta F \wedge F=\delta * F \wedge * F=\varepsilon \frac{1}{6} \operatorname{Tr}\left[\odot\left(\Psi, * \mathbf{d} \Psi^{*}\right)\right]
\end{aligned}
$$

where $\delta$ is the coderivatie. Denoting by $|\mathcal{R}|^{2}$ the quantity $\frac{1}{6}|* \operatorname{Tr}[\odot(\mathcal{R} \wedge * \overline{\mathcal{R}})]|$ we obtain (in the $F$-adapted coordinate system)

$|\mathcal{R}|^{2}=\frac{1}{6}|* \operatorname{Tr}[\odot(\mathbf{d} \Psi, * \mathbf{d} \bar{\Psi})]|=\left(u_{\xi}-\varepsilon u_{z}\right)^{2}+\left(p_{\xi}-\varepsilon p_{z}\right)^{2}=|\delta F|^{2}=|\delta * F|^{2}$.

Finally we note the relations

$$
\frac{1}{6} \operatorname{tr}(\chi)=\frac{1}{6} \operatorname{tr}(u \mathcal{I}+p \mathcal{J})=u
$$

and

$$
\frac{1}{6} \operatorname{tr}[(\chi \circ \bar{\chi})]=\frac{1}{6} \operatorname{tr}[(u \mathcal{I}+p \mathcal{J}) \circ(u \mathcal{I}-p \mathcal{J})]=u^{2}+p^{2}
$$


These relations allow to introduce two characteristic functions for any nonlinear solution: the phase-function $\psi$ and the scale factor $\mathcal{L}$ as

$$
\psi=\arccos \frac{\frac{1}{6} \operatorname{tr} \chi}{\sqrt{\frac{1}{6} \operatorname{tr}(\chi \circ \bar{\chi})}}, \quad \mathcal{L}=\frac{\sqrt{\frac{1}{6} \operatorname{tr}(\chi \circ \bar{\chi})}}{\sqrt{\frac{1}{6}}|\mathcal{R}|}=\frac{\sqrt{\operatorname{tr}(\chi \circ \bar{\chi})}}{|\mathcal{R}|} .
$$

Since the nonlinear solutions of the two equations

$$
\delta F \wedge * F=0, \quad \delta * F \wedge F=0
$$

i.e., those satisfyiing $\delta F \neq 0$ and $\delta * F \neq 0$, are parametrized by two functions $(u, p)$ and satisfy the relations

$$
u\left(u_{\xi}-\varepsilon u_{z}\right)+p\left(p_{\xi}-\varepsilon p_{z}\right)=0, \quad u_{\xi}-\varepsilon u_{z} \neq 0, \quad p_{\xi}-\varepsilon p_{z} \neq 0
$$

in the corresponding $F$-adapted coordinate system, we obtain that on those solutions the following relation holds

$$
\operatorname{Tr}[\odot(\bar{\Psi}, * \mathbf{d} \Psi)]=0
$$

and that the equation

$$
\delta F \wedge F=\delta * F \wedge * F
$$

is equivalent to

$$
\operatorname{Tr}\left[\odot\left(\Psi, * \mathbf{d} \Psi^{*}\right)\right]=\varepsilon \operatorname{Tr}\left[\odot\left(\Psi^{*}, * \mathbf{d} \Psi\right)\right] .
$$

It can easily be shown (we leave this to the reader) that the non-zero value of the squared curvature invariant $|\mathcal{R}|^{2}$ guarantees availability of rotational component of propagation.

\section{Conclusion}

The linear connection $\nabla$ is defined through $\zeta \otimes \rho^{\prime}(\alpha(u, p))$. We note that the two-form $F_{o}=\mathrm{d} x \otimes \zeta$ gives the possibility to consider a nonlinear solution $F(u, p)$ as an appropriately defined linear map

$$
\rho^{\prime}(\alpha(u, p))=u \mathcal{I}+p \mathcal{J}
$$

in $\Lambda^{2}(M)$, since the action of $\rho^{\prime}(\alpha(u, p))$ on $F_{o}$ gives $F$. 
This special importance of $\zeta$ is based on the fact that it intrinsically defines the translational part of the dynamical befavior of the solution, and its uniqueness is determined by the fact that all nonlinear solutions of EED equations have zero invariants: $F_{\mu \nu} F^{\mu \nu}=F_{\mu \nu}(* F)^{\mu \nu}=0$. As for the rotational part of the dynamical behavior of the solution it is available only if the curvature $\mathcal{R}$ iz nonzero and is locally represented by any of the two three-forms $F \wedge \delta F=$ $* F \wedge \delta * F \neq 0$. For all nonlinear solutions we have $\delta F \neq 0$ and $\delta * F \neq 0$, and all finite nonlinear solutions have finite energy density

$$
0<\phi^{2}=\frac{1}{6} \operatorname{tr}(F \circ \bar{F})=\frac{1}{6} \operatorname{tr}(\chi \circ \bar{\chi})=\left(u^{2}+p^{2}\right)<\infty .
$$

The nonzero finite scale factor $0<\mathcal{L}<\infty$ separates those finite nonlinear solutions which carry spin momentum, and this happens only when $|\delta F|=|\mathcal{R}| \neq 0$. The spin momentum is carried by any of the two three-forms $\delta F \wedge F=\delta * F \wedge * F$, determining the energy-momentum exchange between $F$ and $* F$. Clearly, on the linear Maxwell solutions these three-forms are zero.

Hence, in terms of curvature we can say that the nonzero curvature invariant $|\mathcal{R}|$, is responsible for availability of rotational component of propagation, in other words, the spin properties of a nonlinear solution require non-zero curvature.

From physical viewpoint the corresponding dynamical process that generates these spin properties is the mutual energy-momentum exchange between the two field components $F$ and $* F$ during propagation. It has the following three characteristic properies:

- it is permanent, i.e., it occurs constantly during propagation

- it is simultanious in the both directions: $F \rightleftarrows * F$

- it is in equal quantities.

It follows that $F$ and $* F$ live in a permanent dynamical equilibrium. They carry always the same quantities of energy-momentum

$$
\left[F_{\mu \nu} F^{\mu \nu}=0\right] \Rightarrow F_{\mu \sigma} F^{\nu \sigma}=(* F)_{\mu \sigma}(* F)^{\nu \sigma} .
$$

This dynamical equilibrium is quantitatively described by the equation

$$
\delta F \wedge F=\delta * F \wedge * F
$$

or by

$$
\operatorname{Tr}\left[\odot\left(\Psi, * \mathbf{d} \Psi^{*}\right)\right]=\varepsilon \operatorname{Tr}\left[\odot\left(\Psi^{*}, * \mathbf{d} \Psi\right)\right] .
$$




\section{References}

[1] Donev S. and Tashkova M., Extended Electrodynamics: A Brief Review, LANL e-print: hep-th/0403244; From Maxwell Stresses to Photon-like Objects Through Frobenius Curvature Geometrization of Local Physical Interaction, LANL e-print: math-ph/0902.3924.

[2] Greub W., Halperin S. and Vanstone R., Connections, Curvature and Cohomology vol.2, Academic Press, New York, 1973.

Stoil Donev

Institute for Nuclear Research and Nuclear Energy

Bulgarian Academy of Sciences

Blvd. Tzarigradsko chaussee 72

1784 Sofia, BULGARIA

E-mail address: sdonev@inrne.bas.bg

Maria Tashkova

Institute for Nuclear Research and Nuclear Energy

Bulgarian Academy of Sciences

Blvd. Tzarigradsko chaussee 72

1784 Sofia, BULGARIA

E-mail address: mtashkova@inrne.bas.bg 